\title{
Ataxia rating scales in the balance
}

\author{
Stefan M Pulst
}

Remarkable progress has been made towards understanding the molecular and cellular causes of chronic progressive ataxias, yet these conditions are among the last movement disorders to be subjected to controlled treatment trials. This applies not only to neuroprotective treatments, but also to symptomatic treatments. Patients with ataxia could potentially benefit greatly from symptomatic improvement of gait disorders and motor incoordination, even if the progression of the underlying disorder cannot be halted.

A prerequisite for conducting therapeutic trials is the availability of reliable and practical clinical rating scales or performance measures. Until recently, the most widely used scale was the lengthy International Cooperative Ataxia Rating Scale (ICARS). Now, as discussed by Subramony and Sudarsky in the Practice Points section of this issue, two groups have examined additional ways to measure ataxia severity in differing, but potentially complementary, fashions.

Lynch et al. ([2006] Neurology 66: 1711-1716) examined patients with Friedreich ataxia, one of the most common forms of ataxia, and correlated quantitative performance measures with disease duration and disability. They examined timed gait, peg-board performance and low-contrast letter acuity individually and in combination. SchmitzHübsch et al. ([2006] Neurology 66: 1717-1720) developed a novel ataxia rating scale (Scale for the Assessment and Rating of Ataxia; SARA) for patients with autosomal dominant ataxias and validated it by comparison with disease severity and disease duration.

These investigations are crucial, but some important questions remain. What is the natural history and progression of ataxias as measured by these rating scales? How sensitive are these scales at different stages of the disease? Also, are the scales linear in their ability to capture the disease process without showing a ceiling effect? The answers to these questions will be essential for the planning of clinical trials.

\section{A prerequisite for conducting therapeutic trials is the availability of reliable and practical clinical rating scales or performance measures}

SM Pulst is Director of the Division of Neurology and holder of the Carmen and Louis Warschaw Chair in Neurology at Cedars-Sinai Medical Center, Professor of Medicine and Neurobiology at the David Geffen School of Medicine, University of California, Los Angeles, CA, USA, and an Advisory Board member of Nature Clinical Practice Neurology.

\section{Competing interests \\ The author declared he has no competing interests.}

www.nature.com/clinicalpractice doi:10.1038/ncpneuro0446
Disease progression might be overestimated because clinicians are most likely to encounter more-severely affected patients and those patients who return to the clinic more frequently because of rapidly advancing disease. In support of this view, a recent study (Fahey MC et al. [2006] J Neurol Neurosurg Psychiatry [doi: 10.1136/jnnp.2006.096008]) indicated that changes in the Friedreich Ataxia Rating Scale (FARS) score were actually quite small over a 12-month period. Both the underlying rate of progression and the ability of the respective scale to capture it will influence sample sizes in future drug trials.

Compared with Parkinson's disease, spinocerebellar ataxias are more heterogeneous, not only regarding genetic etiologies, but also with respect to involvement of other systems outside the cerebellum. Future research will need to address whether ataxia scales or performance measures provide good composite scores, or whether they are too sensitive to dysfunction in one specific neuronal system. Ataxia rating scales might also face other unexpected challenges. The impaired cerebellum still has the ability to learn motor tasks or improve on already learned tasks. Motivated patients might practice the peg-board test or a timed gait, thereby improving performance on subsequent visits to the clinic despite progression of the underlying disorder. Practice effects will be especially noticeable in short follow-up periods.

Similar to the development of the Unified Parkinson Disease Rating Scale (UPDRS) we can envisage a future 'unified' ataxia rating scale combining features from several rating scales that measure different nonoverlapping aspects of the disease. Furthermore, one can envision use of the SARA, evaluated in dominant ataxias, for measuring progression in Friedreich ataxia. Ultimately, the question of which rating scale is the most appropriate for a given application will only be answered by empirical research. 